\title{
Icosahedral Quasicrystal Layer Observed on $\lambda$ Phase in Al-Cu-Fe Alloy
}

\author{
Andrew Baker'1, Matthew Caputo², Helen Hampikian1, Luke Simpson'1, Chunfei Li1 ${ }^{*}$ \\ ${ }^{1}$ Clarion University of Pennsylvania, Clarion, PA, USA \\ ${ }^{2}$ Youngstown State University, Youngstown, OH, USA \\ Email: *cli@clarion.edu
}

How to cite this paper: Baker, A., Caputo, M., Hampikian, H., Simpson, L. and Li, C.F. (2017) Icosahedral Quasicrystal Layer Observed on $\lambda$ Phase in Al-Cu-Fe Alloy. Materials Sciences and Applications, 8, 509-520. https://doi.org/10.4236/msa.2017.87035

Received: May 12, 2017

Accepted: June 25, 2017

Published: June 28, 2017

Copyright (c) 2017 by authors and Scientific Research Publishing Inc. This work is licensed under the Creative Commons Attribution International License (CC BY 4.0).

http://creativecommons.org/licenses/by/4.0/

\section{cc) (i) Open Access}

\begin{abstract}
Icosahedral quasicrystals display irregular shape if it is embedded in bulk material. If it has free surface, it has well-defined facets, reflecting its unique 5-, 3 -, and 2-fold rotational symmetries. In this study, an $\mathrm{Al}-\mathrm{Cu}-\mathrm{Fe}$ alloy with nominal composition of $\mathrm{Al}_{65} \mathrm{Cu}_{20} \mathrm{Fe}_{15}$ was prepared by arc melting and the microstructure was studied by using Scanning Electron Microscope, Energy Dispersive X-ray Spectroscopy, and Electron Back Scattering Diffraction (EBSD). On the surface of $\lambda$ crystalline phase, an extra layer is found. EBSD from this layer revealed 5-, 3-, and 2-fold rotational symmetries, demonstrating the icosahedral quasicrystalline structure. Further, it has been found that the icosahedral quasicrystalline extra layer and the $\lambda$ substrate have orientation relationship revealed by the coincidences of Kikuchi bands and poles on the EBSD patterns. This report is important to future studies regarding the formation of icosahedral quasicrystalline phase and thin film preparation related to icosahedral quasicrystalline phase.
\end{abstract}

\section{Keywords}

Icosahedral Quasicrystal, Orientation Relationship, Electron Back Scattering Diffraction, Interface

\section{Introduction}

Stable icosahedral quasicrystals (IQC) were discovered in an Al-Cu-Fe alloy by Tsai et al. in 1987 [1]. This particular Al-Cu-Fe alloy has several advantages such as low cost for mass production and it can be prepared relatively easily by arc melting. In 1993, Balzuweit et al. conducted a study investigating various phases of $\mathrm{Al}-\mathrm{Cu}-\mathrm{Fe}$ alloy prepared by arc-melting [2]. They reported a $\lambda_{2}$ phase with column-shaped morphology. This phase is called $\lambda$ phase in later reports and the present paper. It has monoclinic crystal structure [2] [3] with unit cell dimen- 
sions of $\mathrm{a}=1.558 \mathrm{~nm}, \mathrm{~b}=0.796 \mathrm{~nm}, \mathrm{c}=1.251 \mathrm{~nm}$, and $\beta=108.14^{\circ}$. This phase is believed to play an important role in the IQC phase formation [4] [5]. It is worth to mention that Balzuweit et al. further reported that the $\lambda$ phase does not have a smooth surface. In some regions, extra layers were observed. No detailed discussion about the crystal structure of the extra layer and its relationship to the IQC phase formation was given.

IQC phase has been reported to display irregular bulky shape [6] [7], typically for the case when the grains are embedded in an alloy matrix. If the IQC grains have free surface, decahedral pentagonal shape [8] [9], and rhombic triacontahedron [10] [11] shapes are observed. These morphologies reflect the 5-, 3-, and 2 -fold rotational symmetries. There had been efforts to prepare IQC thin films on some substrata with limited success [12] [13] [14] [15] [16].

This paper reports the observation of extra layer on the surface of columnshaped $\lambda$ phase in Al-Cu-Fe alloy with $\mathrm{Al}_{65} \mathrm{Cu}_{20} \mathrm{Fe}_{15}$ nominal composition. It has been shown clearly that this extra layer has IQC structure, which is different from the $\lambda$ phase of the substrate. Further, it has been revealed that the two phases have orientation relationship.

\section{Materials and Methods}

The alloy was prepared by arc melting pure Aluminum (99.99\%), Copper (99.9\%) and Iron (99.9\%) from Good fellow with nominal composition of $\mathrm{Al}_{65} \mathrm{Cu}_{20} \mathrm{Fe}_{15}$. The vacuum chamber was evacuated to a vacuum of $10^{-5}$ torr, purged with pure Ar gas, and finally refilled with Ar gas. The alloy was cast into a water-cooled copper crucible. Therefore, the cooling rate at the bottom of the ingot is higher than on the free surface. The hemisphere shaped alloy ingot has a diameter of two centimeter and height of one centimeter. The as-prepared alloy was then fractured and the naturally exposed surfaces are examined by using a TESCAN Vega-3 XMU Scanning Electron Microscope (SEM) with secondary electron detector. The electron beam is accelerated with a voltage of $30 \mathrm{kV}$. Elemental analysis was carried out using Oxford Aztec Energy Dispersive X-ray Spectrometer (EDS). Electron Back Scattering Diffraction (EBSD) was done using an OxfordNordlysMax3 EBSD detector. X-ray diffraction (XRD) was performed on a Bruker-NoniusD8 Advance Powder Diffractometer with copper $k_{\alpha}$ line.

\section{Results and Discussion}

Figure 1 is a combination of two SEM images showing three types of grains with distinct morphologies observed in the alloy. They are column-like structures in the center of the main image, spherical particles and grains with distinct pentagonal facets in the inlet. In previous studies [2] [4] [5], the spherical particles have been identified as $\beta$ phase, the column-like structure as $\lambda$ phase, and the particles with distinct pentagonal facets as IQC phase. XRD analysis was carried out to confirm such phase identification. XRD's from different several places were collected and shown in Figure 2 to ensure a representative sampling. Peaks 


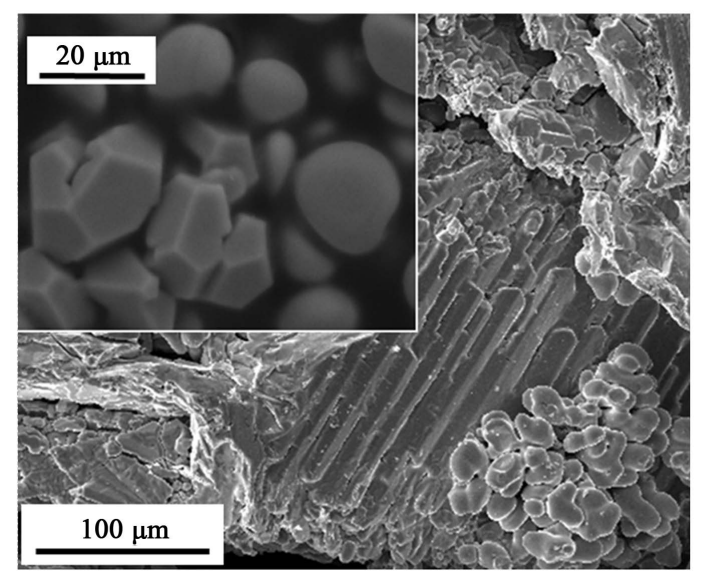

Figure 1. SEM images showing particles with three distinct morphologies. The central part of the main image shows column-like structures while particles with spherical shape and distinct pentagonal facets are observed in the inlet.

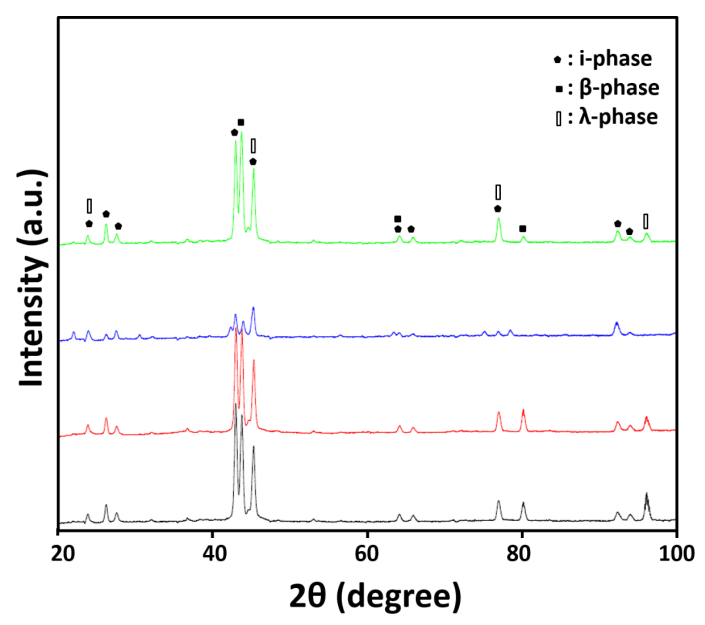

Figure 2. XRD patterns from four different areas of the present alloy.

correspond to these three phases are identified, confirming the above phase identification. As shown in Figure 3, a detailed SEM observation of the columnlike structure revealed that the surfaces are not smooth. There are places where the substrate is exposed while in others the substrate is covered with extra layer. EBSD patterns taken from the substrates of adjacent column-like structures are the same and a typical one is shown in Figure 4(a). This implies that these adjacent column-like structures are parts of a single crystal. Two types of EBSD patterns are observed from the extra layer and are shown in Figure 4(b) and Figure 4(c), respectively. They are different from that in Figure 4(a). As to be explained in detail in the next paragraph, despite their similarities in the layout of Kikuchi bands, (b) and (c) are featured by 5-, 3-, and 2- fold rotational symmetries while (a) lacks of such symmetries. Further, the Kikuchi bands, such as those marked as I-3's, located approximately in the same place in the EBSD patterns have different widths. These are considered as due to the different structure from the substrate to the extra layer. Some of the locations where the EBSD patterns were taken are marked on Figure 3. It follows that if the crystalline structure is 


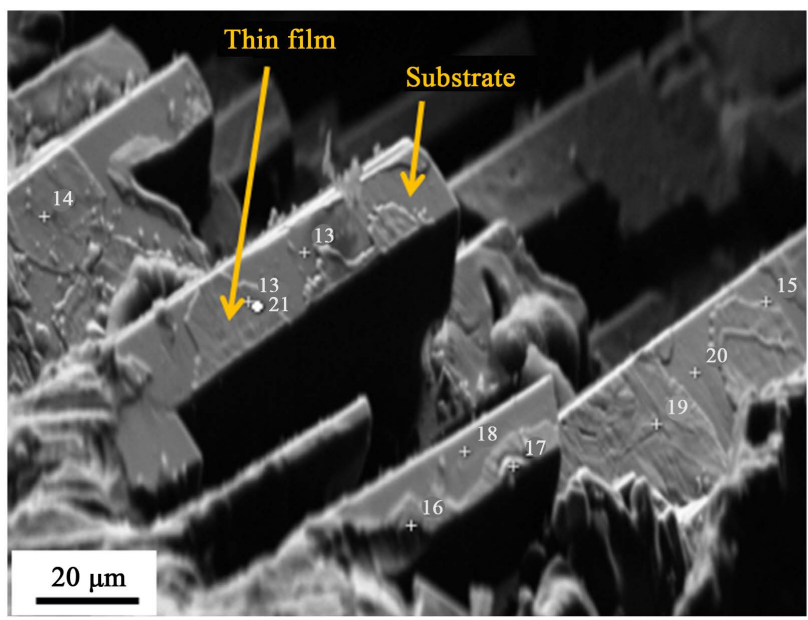

Figure 3. An enlarged SEM image of a column-like structure. Areas of substrate and extra layer are marked. Areas marked with numbers represent places from where EBSD patterns were taken.
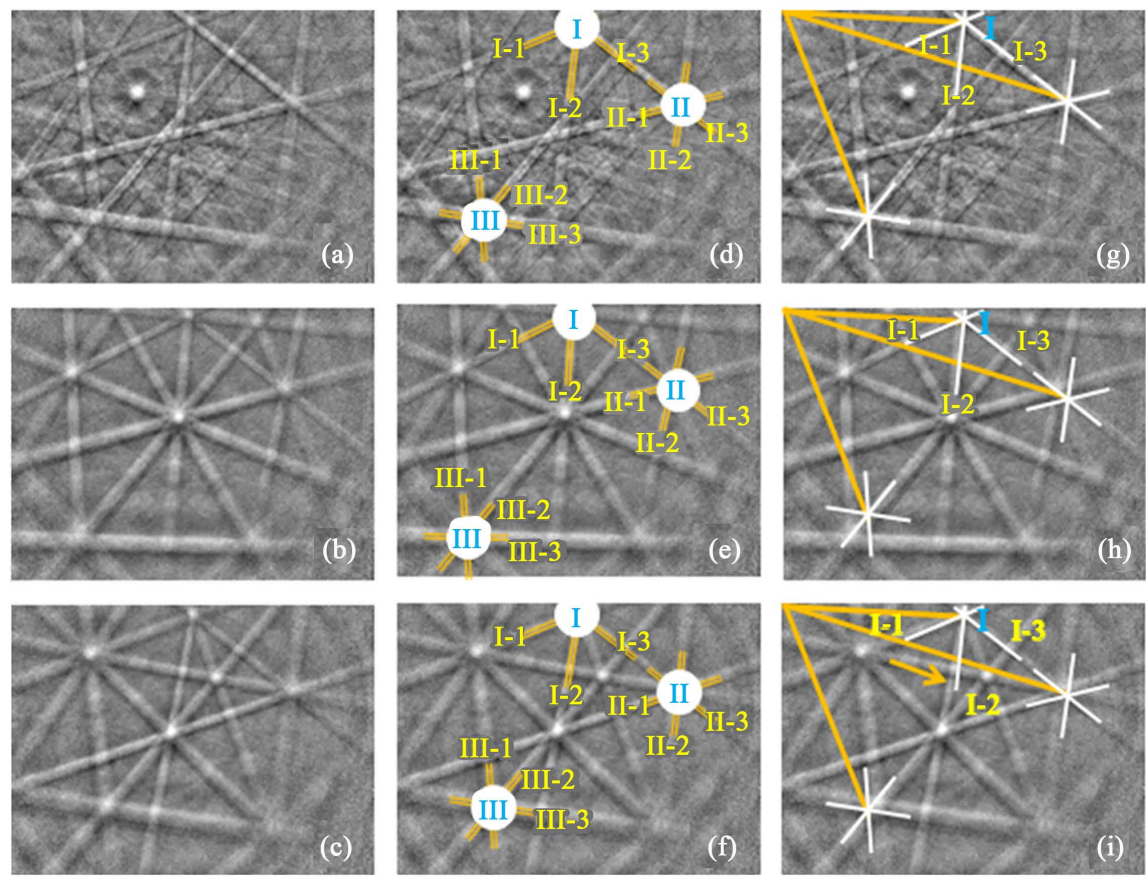

Figure 4. Representative EBSD patterns taken from the substrate (a) and extra layers (b) and (c) of column-like structures shown in Figure 3. The next two columns, (d) to (f) and (g) to (i), are copies of column (a) to (c) with extra drawings on top. Despite the overall difference of the three patterns, there are similarities, which are illustrated in the column of (d) to (f) by adding trace drawings of similar Kikuchi bands and poles. For example, in (d), Kikuchi bands I-1, I-2, and I-3 intercept with each other on pole I. This set of Kikuchi bands and pole have their similar counterparts in both (e) and (f). A detailed examination of the similarities is provided in column (g) to (i). Short lines are drawn to represent the Kikuchi bands and long lines were drawn from the left upper corner to the intercepts of the short lines to represent the positions of the sets in (g). These drawings are copied, pasted in (h) and (i), and are placed in such a way that the shared end of the long position lines is on the left upper corner. The degree of overlapping of Kikuchi bands and pole sets in (g) with those in (h) and (i) can be evaluated in terms of position of the poles and the orientation of the bands. 
different for the substrate and the extra layer, there may also be a difference in composition. Result of EDS elemental mapping on an area containing both substrate and extra layer is shown in Figure 5. The central part of the image enclosed with whilte dotted lines corresponds to the substrate. It is interesting to note that the IQC extra layer on the left is not flat, resembling a step-like feature. The contrast changes going from the substrate to the extra layer in all three elemental mapping images.

It is concluded that the extra layer has an IQC structure based on the following analysis. Figure 6 shows the two types of EBSD patterns from the extra layer again, where Figure 6(a) corresponds to the pattern of Figure 4(b) and Figure $6(\mathrm{~b})$ to that of Figure 4(c). On top of these patterns, the corresponding positions of 5-, 3-, and 2-fold rotational symmetry axes are marked. It is well-known that 5-fold rotational symmetries are not permitted in regular crystalline structure. The co-existence of 5-, 3-, and 2-fold rotational symmetries are unique for IQC phase. If the specimen is tilted correctly and the EBSD pattern has sufficient large field of view, these symmetry axes on EBSD pattern are arranged in the
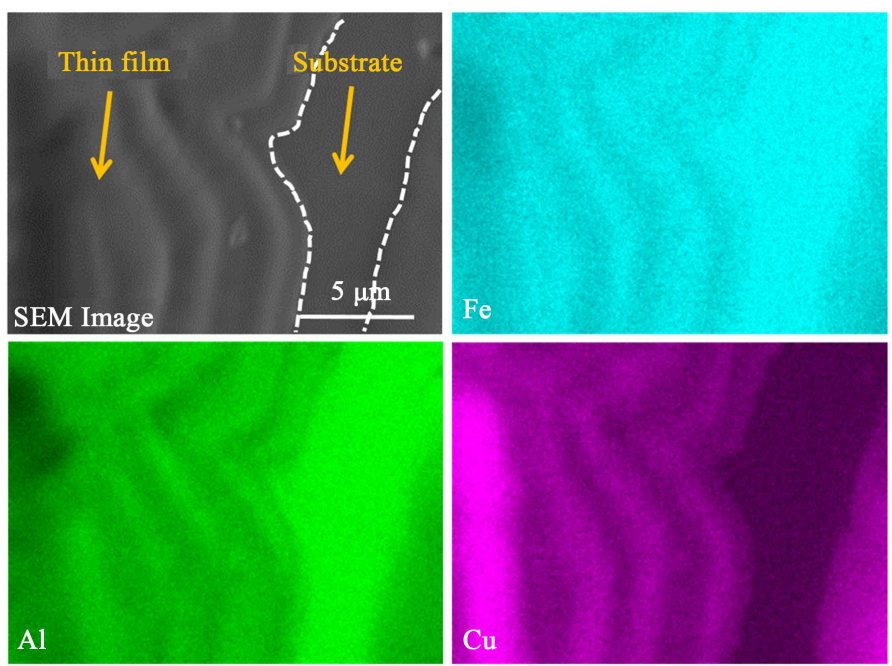

Figure 5. SEM and elemental mapping images of a section of column-like structure.

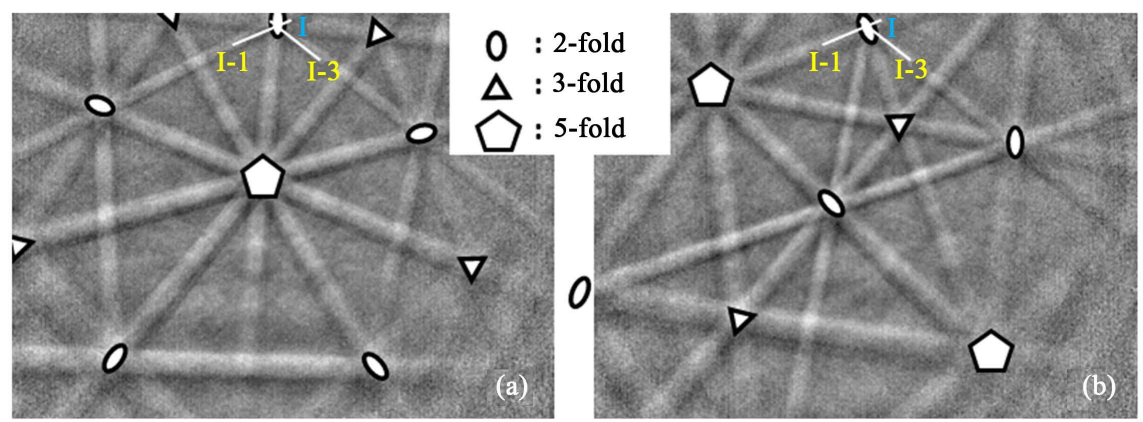

Figure 6. Two typical EBSD patterns from extra layer of Figure 3. Pole positions corresponding to 5-, 3-, and 2-fold rotational symmetry axes are marked. Although both (a) and (b) are from IQC phase, they have different orientation. Trace drawings of the two Kikuchi bands and one pole, which are important in determining the orientation relationship between the IQC extra layer and $\lambda$ substrate, are added. 
following way. If you consider the point corresponding to 5 -fold rotational symmetry axis is in the center, there are five points related to2-fold rotational symmetry axes arranged with symmetry, forming small pentagon. Further out, there are five points related to 3 -fold rotational axes arranged in a similar way, forming a larger pentagon. Such relative arrangement of the rotational axes is consistent with IQC structure and previous reports [17] [18], proving clearly that the extra layer is of IQC structure, different from the substrate $\lambda$ phase. Further, the result of elemental mapping supports this conclusion. The columnlike structure has been identified as $\lambda$-phase (or $\omega$-phase) with chemical formula of $\mathrm{Al}_{13} \mathrm{Fe}_{4}$ [4] [5] or $\mathrm{Al}_{7} \mathrm{Cu}_{2} \mathrm{Fe}$ [19] [20] in previous reports. This chemical formula corresponds to an atomic concentration of $\mathrm{Al}_{77} \mathrm{Fe}_{23}$ or $\mathrm{Al}_{70} \mathrm{Cu}_{20} \mathrm{Fe}_{10}$. The $\mathrm{Al}_{70} \mathrm{Cu}_{20} \mathrm{Fe}_{10}$ phase is also known as $\omega$ phase. The IQC phase is reported to have a composition of $\mathrm{Al}_{65} \mathrm{Cu}_{20} \mathrm{Fe}_{15}$ [11]. As shown in Figure 5, compared to the substrate, the extra layer shows clear decrease in Aluminum and Iron concentrations, and an increase in Copper concentration, which is consistent with the nominal compositions of the $\lambda$ and IQC phases.

Comparison of the EBSD patterns from the substrate and IQC extra layer revealed orientation relationship between the two. A close examination of the EBSD patterns shown in Figure 4(a) from $\lambda$ substrate and Figure 4(b) and Figure 4(c) from extra layers revealed some similar features, despite their different crystal structure. To explain the similarity, the original EBSD patterns shown in the left column of Figure 4 are shown two more times in the next two columns. In the second column of Figures 4(d)-(f), the seemingly common features are emphasized by trace drawings on top of each pattern. For example, Kikuchi bands I-1, I-2, and I-3 intercept with each other at point I in Figure 4(d). The intercepting points correspond to crystalline/quasi crystalline poles. Each set of three Kikuchi bands and one pole in Figure 4(d) can find their counterparts in EBSD patterns of Figure 4(e) and Figure 4(f) from extra layer. A more detailed examination revealed that the similarities between Kikuchi bands named as I-1 and I-3, and pole I are more consistent than others, and hence is considered to play more important role in defining the orientation relationship. This is demonstrated in the third column of Figures 4(g)-(i). In Figure 4(g), the position of the pole I is represented by a long line drawn from the left upper corner to it. The Kikuchi bands of interest passing through the other end of the long line were traced with short lines. Similar drawings were done regarding other two sets of Kikuchi bands and poles. Copies of these drawings from Figure 4(g) are placed on top of Figure 4(h) and Figure 4(i). The drawings are placed in such a way that the shared end of the long lines is placed on the left upper corner of the frame, as in the case of Figure $4(\mathrm{~g})$. This way, the overlapping of the copied trace drawings and the Kikuchi band and pole sets can be evaluated in terms of pole position and band orientation. A close examination of Figure $4(\mathrm{~h})$ and Figure 4(i) found that the drawings related poles II and III do not overlap precisely with their corresponding features. The overlapping related to pole I position is good. However, it was found that the line representing I-2 Kikuchi band 
in Figure 4(g) does not overlap with its counterpart features in Figure 4(h) and Figure 4(i) well. This is especially true on Figure 4(i), as marked by an arrow. These results and analysis indicate that the substrate $\lambda$ phase and the extra layer has an orientation relationship defined by the coincidence of Kikuchi bands I-1 and I-3 and pole I on EBSD patterns. It is worthy to point out that, for both types, the shared pole corresponds to the 2 -fold rotational axis.

Two types of orientation relationship between the $\lambda$ substrate and the IQC extra layer are found. As mentioned previously, adjacent column-like structures are parts of a single crystal of $\lambda$ phase. As a result, EBSD patterns from different area of substrate in Figure 3 are the same. In contrast, two types of EBSD patterns from the extra layer are observed, although both of them correspond to IQC phase. The first type is represented by the EBSD pattern shown in Figure 4(b), Figure 4(e), Figure 4(h), and Figure 6(a) while the second by EBSD pattern in Figure 4(c), Figure 4(f), Figure 4(i), and Figure 6(b). The difference can be seen clearly in Figure 6(a) and Figure 6(b). As shown in Figure 6(a), for the first type, theI-1 and I-3 Kikuchi bands from the substrate coincide with two adjacent sides of the small pentagon in the IQCEBSD pattern. In case of type II, as shown in Figure 6(b), I-1 corresponds to the Kikuchi band that passes through the 2- and 5-fold rotational axis points and I-3 corresponds to the Kikuchi band that passes two adjacent 2 -fold rotational axis points.

Such orientation relationship seems to be universal since studies on other areas obtained similar results. As an example, Figure 7 shows a SEM image of another column-like structure. The corresponding EBSD patterns from the substrate and the extra layer are shown in Figure 8(a) and Figure 8(b), respectively. Part of the pattern is blocked because of the rough surface. Figure $8(\mathrm{c})$ shows the same EBSD pattern of Figure 8(a) with trace drawings of important pole and Kikuchi bands, which are considered to be important in defining the orientation

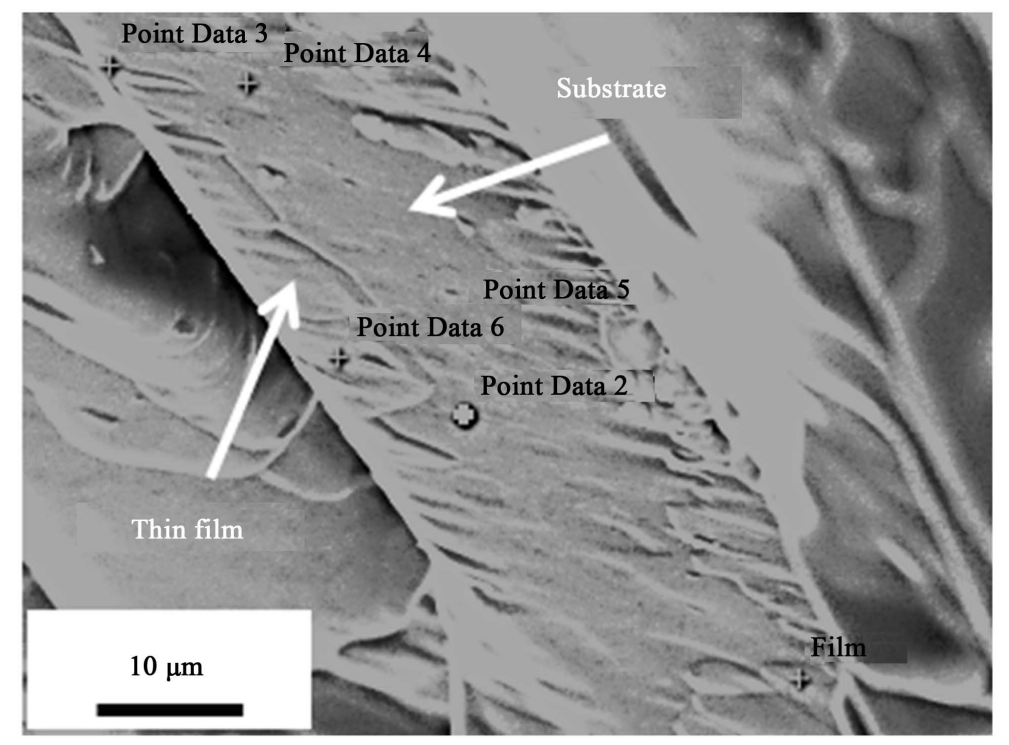

Figure 7. SEM image showing another column-like structure with both substrate and IQC extra layer. 

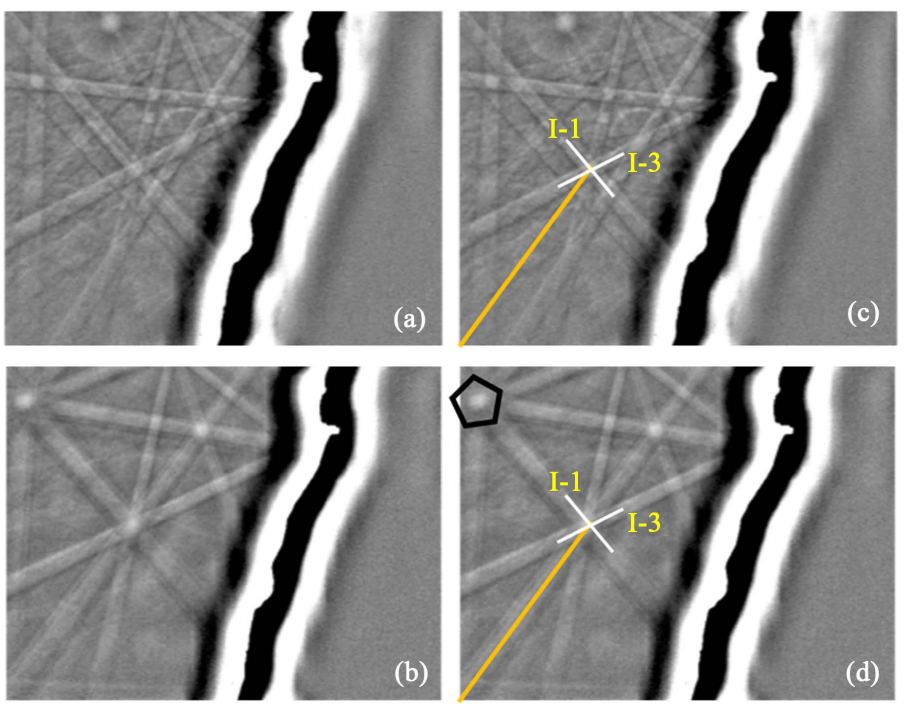

Figure 8. EBSD patterns from substrate (a) and extra layer (b) of the column-like structure shown in Figure 7. The right column displays the same patterns with drawing on top for analysis purpose.

relationship between the substrate and extra layer. In Figure 8(d), the EBSD pattern of Figure $8(\mathrm{~b})$ was shown again with the copied trace drawings from Figure $8(c)$ on top of it. Similar analysis as we have done before leads to the conclusion that the substrate $\lambda$ phase and the extra IQC layer have type II orientation relationship. The shared pole I in Figure 8(d) corresponds to a 2-fold rotation symmetry axis of IQC structure.

It is believed that such orientation relationship results from lowered interface energy. If both the substrate and the extra layer are of crystalline structure, the Kikuchi bands would correspond to lattice planes. The fact that similar Kikuchi bands appear on the EBSD patterns from both the substrate and the extra layer implies a good matching in these lattice planes, typically in the form of epitaxial growth. The driving force for this epitaxial growth is the lowered interface energy because of the improved matching in atomic sites. There is no such concept as lattice planes with IQC phase due to the lack of translational symmetry. However, it is reasonable to consider that certain orientation relationship allows better atomic site matching between substrate and IQC extra layer, and hence reduced interface energy. It should be pointed out that on the interface of crystal to crystal, usually there is only one type of orientation relationship that corresponds to the lowest interface energy or best atomic site matching. However, in the present crystal to IQC interface, there are, at least, two orientation relationship as discussed in the previous paragraphs. This is probably because the interface is the matching between crystal with translational symmetry and IQC structure without translational symmetry. Therefore, more than one orientation relationships are comparable in term of lowered interface energy. It is worth to mention that, in both cases, the shared pole is the 2 -fold rotation axis.

This orientation relationship explains several experimental observations and is valuable for future studies. First, it explains that the extra layer tends to spread 
relatively large areas. For $\lambda$ phase, it is reasonable to consider that, in atomic level, the arrangement of atoms repeats in a distance comparable to its lattice constant, which is in the order of one nanometer. For IQC phase, we use the size of icosahedron to estimate the "repeating" distance [14]. The size of an icosahedron is less than $1 \mathrm{~nm}$. As shown in Figure 3 and Figure 7, the extralayer spreads over several ten micrometers, well beyond the repeating distance for both phases. It is known that when a thin film is formed on a substrate, its morphology depends on the interface energy. If the interface energy is low, the thin film tends to adhere well on the substrate and spread over large area. If the interface energy is high, the thin film tends to form an island and grow in thickness direction [21]. The fact that the present IQC extra layer spreads over relatively large area is considered as the evidence of lowered interface energy. Second, it helps the studies of preparing IQC related thin film. Icosahedral quasicrystalline alloys are reported to be hard and brittle. The brittleness makes it unsuitable for structural purpose. However, the high hardness makes it an appealing material for coating purpose. There have been some studies to create a quasicrystalline coating [12] [13] [14] [15] [16]. There have also been attempt to grow crystalline thin film on top of IQC surface [21] [22]. In an attempt to grow Aluminum thin film on IQC surface, Cai et al. [22] reported that although Aluminum atoms can find favorable atomic sites at low coverage, lateral growth stops and island forms as coverage increases, probably due to the relatively high interface energy or the lack of long-range atomic site matching. Present report indicates that semi-epitaxial growth of IQC thin film on selected crystalline surface is possible, which is a strategy that can be adopted in future studies. Second, it is observed very often that IQC grains with distinct pentagonal facets line-up with each other and the facets from different grains have coordinated orientation, as shown in Figure 9.

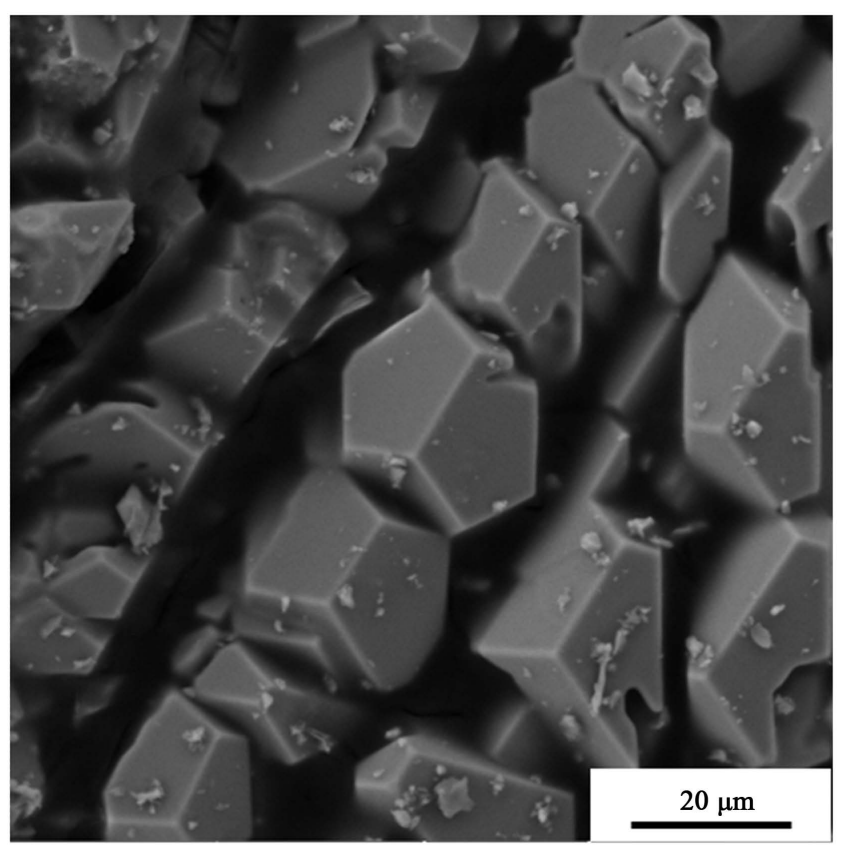

Figure 9. SEM image showing rows of IQC particles with coordinated facet orientation. 
These are explained as the result that the growth of IQC phase uses the column-shaped $\lambda$ phase as template and the IQC phase has orientation relationship with the substrate.

There are several questions remain unanswered regarding the formation of the IQC extra layer. First, it is not clear about the thickness of the interface layer. Is there a transitional layer spreading several atomic layers? Or, the transition is an abrupt switch from one phase to another in atomic level? Second, the orientation relationship between the $\lambda$ substrate and the IQC extra layer is not determined quantitatively in the present work, due to the limited EBSD instrument time. A study of the interface by Transmission Electron Microscopy (TEM) will be helpful to answer some of these questions. Diffraction analysis by TEM can also determine quantitatively the orientation relationship between the substrate and IQC extra layer, the result of which can be used to compare with previous theoretical predictions [23] [24].

\section{Conclusion}

The microstructure of $\mathrm{Al}_{65} \mathrm{Cu}_{20} \mathrm{Fe}_{15}$ alloy prepared by arc melting was studied. Column-shaped $\lambda$ phase, sphere-shaped $\beta$ phase, and IQC particles with well-defined pentagonal facets were observed. The surface of column-shaped $\lambda$ phase was found to be covered with IQC extra layer. EBSD studies revealed that the $\lambda$ substrate and the IQC extra layer have orientation relationship. Different from the epitaxial growth of crystalline thin film on crystalline substrate, there are at least two types of orientation relationships, which are considered to be unique for the interface between crystal and IQC. Lowered interface energy is considered to be the reason for the orientation relationship. A study of the interface by TEM will provide data to have better insight.

\section{Acknowledgements}

Financial support from National Science Foundation (NSF) with Grant No. DMR-1461607 is acknowledged.

\section{References}

[1] Tsai, A.-P., Inoue, A. and Masumoto, T. (1987) A Stable Quasicrystal in Al-Cu-Fe System. Japanese Journal of Applied Physics, 26, L1505. https://doi.org/10.1143/jjap.26.11505

[2] Balzuweit, K., Meekes, H., Tendeloo, G. V. and De Boer, J. L. (1993) On the Relationship between Morphology, Composition and Structure of Al-Cu-Fe Crystals and Quasicrystals. Philosophical Magazine B, 67, 513-532. https://doi.org/10.1080/13642819308207689

[3] Grin, J., Burkhardt, U., Ellner, M. and Peters, K. (1994) Refinement of the Fe4Al13 Structure and Its Relationship to the Quasihomological Homeotypical Structures. Zeitschriftfuer Kristallographie, 209, 479-487. http://cat.inist.fr/?aModele $=$ afficheN\&cpsidt $=4168329$

[4] Cheung, Y.L., Chan, K.C. and Zhu, Y.H. (2001) Characterization of the Icosahedral Phase in As-Cast Quasicrystalline Al $65 \mathrm{Cu} 20 \mathrm{Fe} 15$ Alloy. Materials Characterization, 47, 299-305. https://doi.org/10.1016/S1044-5803(02)00182-1 
[5] Wei, D. and He, Z. (2016) Multilayered Sandwich-Like Architecture Containing Large-Scale Faceted Al-Cu-Fe Quasicrystal Grains. Materials Characterization, 111, 154-161. https://doi.org/10.1016/j.matchar.2015.11.027

[6] Shechtman, D., Blech, I., Gratias, D. and Cahn, J.W. (1984) Metallic Phase with Long-Range Orientational Order and No Translational Symmetry. Physical Review Letters, 53, 1951. https://doi.org/10.1103/physrevlett.53.1951

[7] Daniels, M.J., King, D., Fehrenbacher, L., Zabinski, J.S. and Bilello, J.C. (2005) Physical Vapor Deposition Route for Production of $\mathrm{Al}-\mathrm{Cu}-\mathrm{Fe}-\mathrm{Cr}$ and $\mathrm{Al}-\mathrm{Cu}-\mathrm{Fe}$ Quasicrystalline and Approximant Coatings. Surface and Coatings Technology, 191, 96-101. https://doi.org/10.1016/j.surfcoat.2004.07.117

[8] Guo, J.Q., Sato, T.J., Abe, E., Takakura, H. and Tsai, A.P. (2000) Production of Single Quasicrystals and Their Electrical Resistivity in the Al-Pd-Re System. Philosophical Magazine Letters, 80, 495-502. https://doi.org/10.1080/09500830050057206

[9] Fisher, I.R., Cheon, K.O., Panchula, A.F., Canfield, P.C., Chernikov, M., Ott, H.R. and Dennis, K. (1999) Magnetic and Transport Properties of Single-Grain R-Mg-Zn Icosahedral Quasicrystals [R= $\mathrm{Y},\left(\mathrm{Y}_{1-\mathrm{x}} \mathrm{Gd}_{\mathrm{x}}\right),\left(\mathrm{Y}_{1-\mathrm{x}} \mathrm{Tb} \mathrm{x}_{\mathrm{x}}\right), \mathrm{Tb}, \mathrm{Dy}, \mathrm{Ho}$, and Er]. Physical Review B, 59, 308. https://doi.org/10.1103/PhysRevB.59.308

[10] Dubost, B., Lang, J.M., Tanaka, M., Sainfort, P. and Audier, M. (1986) Large AlCuLi Single Quasicrystals with Triacontahedral Solidification Morphology. Nature, 324, 48-50. https://doi.org/10.1038/324048a0

[11] Tsai, A.P. (2013) Discovery of Stable Icosahedral Quasicrystals: Progress in Understanding Structure and Properties. Chemical Society Reviews, 42, 5352-5365. https://doi.org/10.1039/c3cs35388e

[12] Yoshioka, A., Edagawa, K., Kimura, K. and Takeuchi, S. (1995) Production of HighQuality Thin-Film Samples of Al-Cu-Fe Icosahedral Quasicrystal. Japanese Journal of Applied Physics, 34, 1606. https://doi.org/10.1143/jjap.34.1606

[13] Feng, L.P., Fleury, E. and Zhang, G.S. (2012) Preparation of Al-Cu-Fe-(Sn, Si) Quasicrystalline Bulks by Laser Multilayer Cladding. International Journal of Minerals, Metallurgy, and Materials, 19, 434-440. https://doi.org/10.1007/s12613-012-0575-5

[14] Huttunen-Saarivirta, E. (2004) Microstructure, Fabrication and Properties of Quasicrystalline Al-Cu-Fe Alloys: A Review. Journal of Alloys and Compounds, 363, 154-178. https://doi.org/10.1016/S0925-8388(03)00445-6

[15] Haidara, F., Duployer, B., Mangelinck, D. and Record, M. C. (2012) In-Situ Investigation of the Icosahedral Al-Cu-Fe Phase Formation in Thin Films. Journal of Alloys and Compounds, 534, 47-51. https://doi.org/10.1016/j.jallcom.2012.04.036

[16] Rouxel, D. and Pigeat, P. (2006) Surface Oxidation and Thin Film Preparation of AlCuFe Quasicrystals. Progress in Surface Science, 81, 488-514. https://doi.org/10.1016/j.progsurf.2006.07.002

[17] Lin, C., Hollister, L.S., MacPherson, G.J., Bindi, L., Ma, C., Andronicos, C.L. and Steinhardt, P.J. (2017) Evidence of Cross-Cutting and Redox Reaction in Khatyrka Meteorite Reveals Metallic-Al Minerals Formed in Outer Space. Scientific Reports, 7, Article Number: 1637. https://doi.org/10.1038/s41598-017-01445-5

[18] Asimow, P.D., Lin, C., Bindi, L., Ma, C., Tschauner, O., Hollister, L.S. and Steinhardt, P.J. (2016) Shock Synthesis of Quasicrystals with Implications for Their Origin in Asteroid Collisions. Proceedings of the National Academy of Sciences, 201600321. https://doi.org/10.1073/pnas.1600321113

[19] Dong, C., Dubois, J.M., De Boissieu, M. and Janot, C. (1990) Neutron Diffraction Study of the Peritectic Growth of the $\mathrm{Al}_{65} \mathrm{Cu}_{20} \mathrm{Fe}_{15}$ Icosahedral Quasi-Crystal. Journal of Physics: Condensed Matter, 2, 6339. 
https://doi.org/10.1088/0953-8984/2/30/001

[20] Göğebakan, M., Avar, B. and Uzun, O. (2009) Quasicrystalline Phase Formation in the Conventionally Solidified Al-Cu-Fe System. Materials Science-Poland, 27, 919.

https://scholar.google.com/scholar?q=20.\%09Goebakan+M.\%2C+Uzun+O.\%2C+M ater.+Sci.-Poland\%2C+27+\%282009\%29\%2C+919.\&btnG=\&hl=en\&as_sdt=0\%2C5

[21] Fournée, V. and Thiel, P.A. (2005) New Phenomena in Epitaxial Growth: Solid Films on Quasicrystalline Substrates. Journal of Physics D: Applied Physics, 38, R83. https://doi.org/10.1088/0022-3727/38/6/r01

[22] Cai, T., Ledieu, J., McGrath, R., Fournée, V., Lograsso, T., Ross, A. and Thiel, P. (2003) Pseudomorphic Starfish: Nucleation of Extrinsic Metal Atoms on a Quasicrystalline Substrate. Surface Science, 526, 115-120. https://doi.org/10.1016/S0039-6028(02)02593-1

[23] Barbier, J.N., Tamura, N. and Verger-Gaugry, J.L. (1993) Monoclinic $\mathrm{Al}_{13} \mathrm{Fe}_{4}$ Approximant Phase: A Link between Icosahedral and Decagonal Phases. Journal of Non-Crystalline Solids, 153, 126-131. https://doi.org/10.1016/0022-3093(93)90328-U

[24] Cheng, Y.F., Hui, M.J. and Li, F.H. (1991) An Intermediate State between the Decagonal and Monoclinic Phases in an Al-Cu-Fe Alloy. Philosophical Magazine Letters, 64, 129-132. https://doi.org/10.1080/09500839108214678

Submit or recommend next manuscript to SCIRP and we will provide best service for you:

Accepting pre-submission inquiries through Email, Facebook, LinkedIn, Twitter, etc. A wide selection of journals (inclusive of 9 subjects, more than 200 journals) Providing 24-hour high-quality service User-friendly online submission system Fair and swift peer-review system Efficient typesetting and proofreading procedure Display of the result of downloads and visits, as well as the number of cited articles Maximum dissemination of your research work

Submit your manuscript at: http://papersubmission.scirp.org/

Or contactmsa@scirp.org 